\title{
Non-operative management of abdominal gunshot injuries: Is it safe in all cases?
}

\author{
Nidal iflazoğlu ${ }^{1}$, Orhan Üreyen², Osman Zekai Öner ${ }^{3}$, Ulvi Mehmet Meral ${ }^{4}$, Murat Yülüklü ${ }^{5}$
}

\section{ABSTRACT}

ORCID IDs of the authors: N.I. $0000-0001-7727-602 X$ 0.Z.0.0000-0002-7241-7863

\section{Cite this paper as:} iflazoğlu N, Üreyen 0, Öner $0 Z$, Meral UM, Yülüklü M. Non-operative management of abdominal gunshot injuries: Is it safe in all cases? Turk J Surg 2018; 34: 38-42.

'Department of General Surgery, Kilis State Hospital, Kilis, Turkey

2Department of General Surgery, Bozyaka Training and Research

Hospital, İzmir, Turkey

${ }^{3}$ Department of General Surgery, Antalya Training and Research Hospital, Antalya, Turkey

${ }^{4}$ Department of General Surgery, İzmir Military Hospital, İzmir, Turkey

${ }^{5}$ Department of General Surgery, Afyon State Hospital, Afyonkarahisar, Turkey

This study was presented at the The $50^{\text {th }}$ Congress of the European Society for Surgical Research, 10-13 June 2015, Liverpool, UK.

Address for Correspondence Nidal İflazoğlu

e-mail: nidal1933@yahoo.com

Received: 12.12.2016

Accepted: 25.02.2017

Available Online Date: 04.01.2018

CCopyright 2018

by Turkish Surgical Association

Available online at

www.turkjsurg.com
Objective: In line with advances in diagnostic methods and expectation of a decrease in the number of negative laparotomies, selective non-operative management of abdominal gunshot wounds has been increasingly used over the last three decades. We aim to detect the possibility of treatment without surgery and present our experience in selected cases referred from Syria to a hospital at the Turkish-Syrian border.

Material and Methods: Between February 2012 and June 2014, patients admitted with abdominal gunshot wounds were analyzed. Computed tomography was performed for all patients on admission. Patients who were hemodynamically stable and did not have symptoms of peritonitis at the time of presentation were included in the study. The primary outcome parameters were mortality and morbidity. Successful selective non-operative management (Group 1) and unsuccessful selective non-operative management (Group 2) groups were compared in terms of complications, blood transfusion, injury site, injury severity score (ISS), and hospital stay.

Results: of 158 truncal injury patients, 18 were considered feasible for selective non-operative management. of these, 14 (78\%) patients were treated without surgery. Other Four patients were operated upon progressively increasing abdominal pain and tenderness during follow-up. On diagnostic exploration, all of these cases had intestinal perforations. No mortality was observed in selective non-operative management. There was no statistically significant difference between Group 1 and Group 2, in terms of length of hospital stay (96 and $127 \mathrm{~h}$, respectively). Also, there was no difference between groups in terms of blood transfusion necessity, injury site, complication rate, and injury severity score $(\mathrm{p}>0.05)$.

Conclusion: Decision making on patient selection for selective non-operative management is critical to ensure favorable outcomes. It is not possible to predict the success of selective non-operative management in advance. Cautious clinical examination and close monitoring of these patients is vital; however, emergency laparotomy should be performed in case of change in vital signs and positive symptoms concerning peritonitis.

Keywords: Abdominal, gunshot, non-operative management, selective

\section{INTRODUCTION}

In 1960 , Shaftan (1) reported that $34.1 \%$ of patients with abdominal injury were laparotomy negative and suggested that the need for surgery in abdominal injury patients should be determined with physical examination. In his series, the non-operatively treated patients comprised mostly patients with stab wounds, but included some with gunshot wounds. The negative laparotomy rate of about $20 \%$ determined during the Vietnam War was found acceptable because of the low morbidity in laparotomy negative cases (2). Subsequently, serious objections to obligatory laparotomy were raised, and consequently, a selective conservative approach in penetrating abdominal traumas was introduced to treatment $(3,4)$. In 1986, Demetriades et al. (5) non-operatively treated penetrating hepatic injuries with a success rate of $33 \%$. Objections to this approach peaked in the 1990s. The negative outcomes of negative laparotomy were scrutinized and it was reported that the rates of complications associated with negative laparotomy were about 20 percent (6-9). With advances in diagnostic methods and the expectation of a decrease in the number of negative laparotomies, the obligatory surgical exploration in all penetrating abdominal injuries was questioned. Many authors reported acceptable results with non-operative therapy in patients with no findings related to peritonitis and with hemodynamic stability (8-10). The follow-up procedure of abdominal gunshot injuries has been increasingly used for the past three decades. Selective non-operative management (SNOM), supported by prospective and retrospective studies, is currently a popular approach (11-19). Evidence supporting the practibility and safety of non-operative therapy in abdominal injuries is increasingly being reported $(20,21)$.

The purpose of this study is to investigate feasibility of non-operative therapy in cases with penetrating abdominal gunshot injuries and to present experience of our second-degree center, situated at the Turkish-Syrian border, which receives many gunshot injury referrals from Syria's active battle districts.

\section{MATERIAL AND METHODS}

Patients with penetrating truncal gunshot injuries in the period between February 2012 and June 2014 were retrospectively studied. Of these patients, those who were hemodynamically stable and did not 
have signs and symptoms of peritonitis at the time of presentation were included in the study. Patients who had undergone emergency surgery and with incomplete patient files were excluded from the study.

The data from the patients were retrospectively studied in terms of age, gender, injury site (anterior abdomen or posterior abdomen), number of blood transfusions, abdominal organ injuries (those determined), extra-abdominal organ injuries, length of hospital stay and need for laparotomy after followup. Non-surgically followed patients were divided into two groups as successful SNOM (Group 1) and unsuccessful SNOM (Group 2) according to necessity of surgical treatment during follow-up period.

The area among the line between both nipples and the symphysis pubis was accepted as the anterior abdomen, and the area between the inferior angles of the scapulae and gluteal folds was accepted as the posterior abdomen. The mid-axillary line was used to demarcate the anterior abdomen from the posterior. Patients who were hemodynamically stable, with no signs and symptoms of peritonitis, and with penetrating abdominal injury determined by radiological methods, were selected for SNOM. Hemodynamic stability was accepted as a systolic blood pressure of above $90 \mathrm{~mm} \mathrm{Hg}$ and a pulse under $110 /$ min. Patients without abdominal pain, tenderness, defense, or rebound were accepted to have no peritonitis. All patients underwent abdominal and thoracic computed tomography (CT) during the first admission. When required, cerebral $C T$, extremity $C T$, and direct radiographies were also performed for diagnostic purposes.

The patients' recovery criteria were no abdominal pain, oral intake of food, no vomiting, bowel movement, adequate bowel sounds by auscultation, no fever and tachycardia, and no finding or complaint indicating any pathology. All patients were examined (about every $3 \mathrm{~h}$ ), followed up, and operated by the same surgeon. The patients were discharged from the hospital when their general condition was stable and with their own will. Because of the retrospective nature of our study, there was no need for an ethical committee approval. The study was designed according to "World Medical Association Declaration of Helsinki Ethical Principles for Medical Research Involving Human Subjects," amended in October 2013. The patients participating in the study gave their approval after being informed regarding the possible complications that could occur during therapy and follow-up.

\section{Statistical Analysis}

Statistical Package for the Social Sciences version 22 software package (IBM Corp.; Armonk, NewYork, USA) was used for statistical analysis. For the analysis of categorical variables and continuous data, Fisher's Exact test and Mann-Whitney $U$ test were used, respectively. $\mathrm{p}<0.05$ was considered statistically significant.

\section{RESULTS}

One hundred and fifty-eight cases were analyzed. One hundred and forty patients were operated and negative laparotomy rate was $7 \%(n=11)$ in operatively treated group. Eighteen patients $(11.3 \%)$ who were followed in intensive care unit without surgical intervention were included in the study (Figure 1). The patients were all male and the average age was $29.6( \pm 8.34)$ years; $35.7 \%(n=5)$ of these non-surgically treated patients had radiologically diagnosed solid organ injuries and were managed conservatively. Of these patients, 3, 1 and 1 had liver, spleen, and kidney injuries, respectively. On CT examination in Group 1, apart from solid organ injuries, 3 patients had isolated intraperitoneal foreign bodies, 3 had free air and foreign body, 2 had degradation of peritoneal surface and mesointestinal edema, and 1 had only free air. CT images of 3 cases belonging to Group 1, showing solid organ injury, intra-abdominal extraluminal air, and intra-abdominal foreign body are shown in the Figure 2, 3.

Four (22.2\%) patients underwent surgery because of peritonitis signs during the intensive care follow-up. Besides changes in vital signs, intra-abdominal free air was observed in four
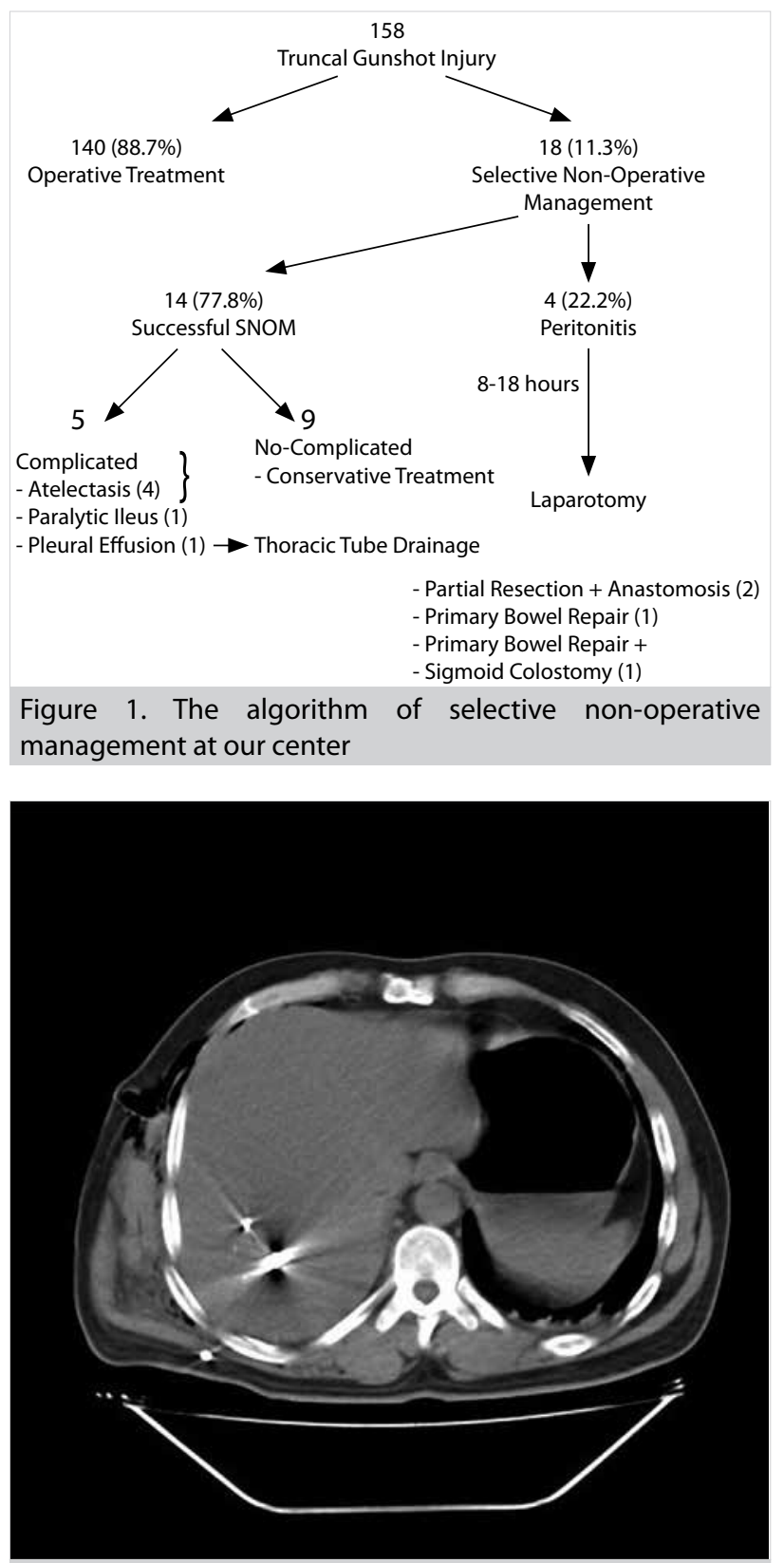

Figure 2. CT scan of a selective non-operative management patient. Foreign bodies are seen in liver parenchyma and right thoracic subdermal space. Intra-abdominal extraluminal air can be seen 
(22\%) patients on CT, and delayed laparotomy was performed for these patients. Patients who were in unsuccessful SNOM (Group 2) group were kept under observation for 13 (8-18) h before surgery. Small bowel injuries were noted in three patients and small bowel-colonic injury was determined in one patient. Partial resection and primary anastomosis in two patients, primary repair of small bowel in one patient, and primary bowel repair+sigmoid colostomy in one patient were performed for the treatment of bowel injuries (Figure 1).

Of the total number of patients, $55.5 \%(n=10)$ had been wounded in the anterior abdomen, $38.8 \%(n=7)$ in the posterior abdomen, and $5.5 \%(n=1)$ in the right anterior hemi thorax. There was no difference between groups in terms of injury site $(p=1.000)$. In the analysis of Group 2 patients with regard

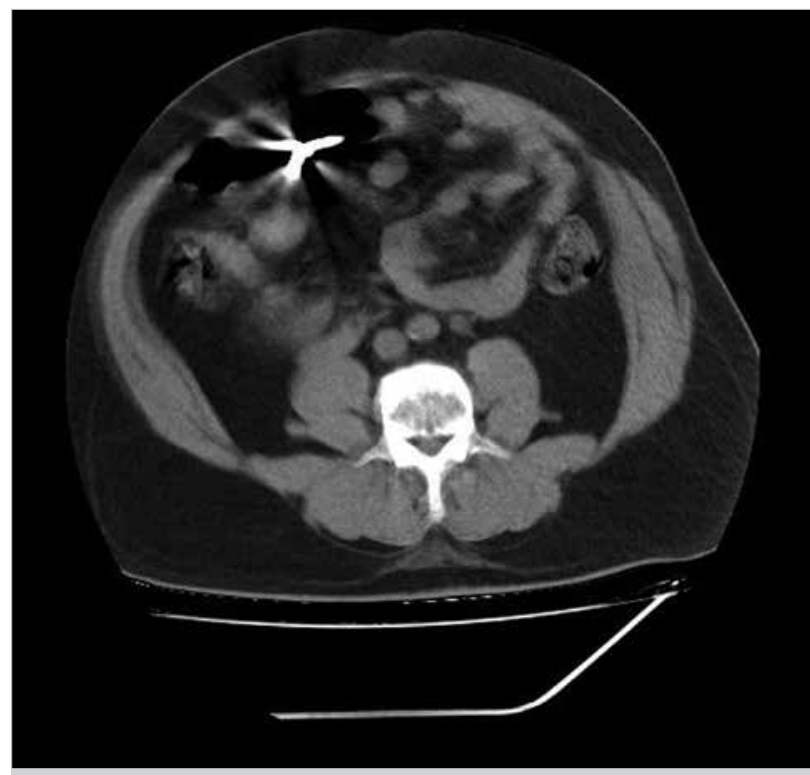

Figure 3. A CT scan of intra-abdominal foreign body in the successful selective non-operative management group to wound site, $50 \%(n=2)$ had been wounded in the anterior abdomen and $50 \%(n=2)$ had been wounded in the posterior abdomen.

There was no mortality in either Group 1 or 2. Total complication rate was $44.4 \%(n=8)$. The rate of complications in Group 1 was $35.7 \%(n=5)$. The complications were lung atelectasis in three patients, paralytic ileus in one patient, and pulmonary effusion in one patient. Although the patients with atelectasis and paralytic ileus were treated with non-surgical approaches, pulmonary effusion was treated with thoracic tube drainage. Surgical site infection developed in three (75\%) Group 2 patients. There was no significant difference between groups in terms of complication rate $(p=0.275)$. There was no significant difference between Group 1 and Group 2, in terms of average injury severity score (ISS) (4.9 and 6, respectively, $p=0.422$ ).

Of the patients, $44.4 \%(n=8)$ did not receive blood transfusion, and of those who did, the average transfusion volume was 1.2 units. There was no significant difference between groups in terms of blood transfusion ( $p=0.129$ ). The average total length of hospitalization was $103 \mathrm{~h}$. There was no significant difference between groups in terms of hospitalization period ( $p=0.202)$. A detailed comparison of Groups 1 and 2 is shown in Table 1. Six (33.3\%) patients in SNOM group also had extra-abdominal organ injuries. Four of them had a pulmonary injury, one had an upper leg injury, and one had a forearm injury. The treatment of pulmonary injury cases involved thoracic tube drainage in three (75\%) and conservative management in one (25\%). Upper leg and forearm injuries treated conservatively. No major surgical intervention was performed in these patients. The distribution of all organ injuries is shown in Table 2.

\section{DISCUSSION}

Selective non-operative management of cases with abdominal gunshot injuries decreases the rate of negative laparotomies. In addition, the non-operative management of penetrating abdominal gunshot wounds means reduced potential for post-

Table 1. Detailed comparison of Group 1 and Group 2 patients

\begin{tabular}{|c|c|c|c|c|c|c|}
\hline & \multicolumn{6}{|c|}{ SNOM } \\
\hline & & \multicolumn{2}{|c|}{ Group 1} & \multicolumn{2}{|c|}{ Group 2} & \multirow[t]{2}{*}{$p$} \\
\hline & & $\mathbf{n}$ & $\%$ & $\mathbf{n}$ & $\%$ & \\
\hline \multirow[t]{3}{*}{ Wound Site } & Anterior & 2 & 50.0 & 8 & 57.1 & \multirow[t]{3}{*}{1.000} \\
\hline & Posterior & 2 & 50.0 & 5 & 35.7 & \\
\hline & With Hemitorax & 0 & 0.0 & 1 & 7.1 & \\
\hline \multirow[t]{2}{*}{ Complication } & + & 3 & 75.0 & 5 & 35.7 & \multirow[t]{2}{*}{0.275} \\
\hline & - & 1 & 25.0 & 9 & 64.3 & \\
\hline \multirow[t]{3}{*}{ Extra-abdominal injury } & + & 0 & 0.0 & 6 & 42.9 & \multirow[t]{2}{*}{0.245} \\
\hline & - & 4 & 100.0 & 8 & 57.1 & \\
\hline & & Mean \pm SD & Min.-Max. & Mean. \pm SS & Min.-Max. & \\
\hline Age & & $28.5 \pm 7.77$ & $19-38$ & $29.93 \pm 8.75$ & $18-50$ & 0.873 \\
\hline Blood Tx Necessity (Unit) & & $0.25 \pm 0.5$ & $0-1$ & $1.5 \pm 1.79$ & $0-6$ & 0.129 \\
\hline Injury severity score (ISS) & & $6 \pm 2.71$ & $4-10$ & $4.93 \pm 3.93$ & $0-12$ & 0.422 \\
\hline Hospital Stay (hour) & & $127 \pm 14$ & $110-142$ & $96.86 \pm 50.38$ & $24-188$ & 0.202 \\
\hline
\end{tabular}


Table 2. Injured organs diagnosed by CT scans and their distribution in patients

\begin{tabular}{|c|c|c|}
\hline Injured organs & SNOM successful & SNOM unsuccessful \\
\hline Abdominal & & 0 \\
\hline Liver & 2 & 0 \\
\hline Kidney & 1 & 0 \\
\hline Spleen & 1 & 0 \\
\hline Colon & 0 & 1 \\
\hline Small bowel & 0 & 4 \\
\hline \multicolumn{3}{|l|}{ Extra-abdominal } \\
\hline Lung & 4 & 0 \\
\hline Femur & 1 & 0 \\
\hline Forearm & 1 & 0 \\
\hline
\end{tabular}

CT: computed tomography; SNOM: selective non-operative management

operative complications, depending on the prevention of negative laparotomies $(7,8)$. The rate of negative laparotomy was reported above $20 \%$ and the rates of complications associated with negative laparotomy were reported to be in the range of $19.7 \%-25.9 \%(6-9)$. In the present study, the rate of negative laparotomy was $7 \%(n=11)$. In our opinion, if we would not apply SNOM for our suitable cases, negative laparotomy rate would increase up to $15.8 \%(n=25)$. We believe that potential complications of surgical interventions were prevented in these patients by following SNOM. Diagnostic laparoscopy can be considered in hemodynamically stable patients to determine peritoneal penetration with potential intestinal injuries and to decrease the number of negative laparotomies (12). Diagnostic laparoscopy in gunshot wounds, in some cases, can lead to false negative results and requires experience in this sense. For our case series, diagnostic laparoscopy was not performed due to insufficient technical capability and low experience.

DuBose et al. (22) reported that non-operative approach is a safe method for appropriately selected patients' solid organ injuries in abdominal gunshot wounds. They presented $9 \%$ SNOM failure with patients having solid organ injuries. In our study, all of five patients who had solid organ injuries were treated with SNOM because of their low-grade injuries. It is crucial for the patients under SNOM to undergo abdominal CT scanning. CT shows whether the injury has penetrated the abdomen or not, the injury path, and the presence of intraabdominal solid and luminal organ injuries (23). In addition, the basic approach should include CT scanning, physical examination, vital findings, and clinical observation, as we did in our study (18-21).

One of the most important problems in the management of abdominal gunshot injuries with SNOM is delayed surgical treatment of luminal organ injuries. SNOM is especially recommended in solid organ injuries (22). The reason of SNOM failure cases was the presence of luminal organ injury. While the clinical examination and radiological findings were normal at admission, the treatment and management changed during the follow-up period by the development of alarming symptoms. These findings emphasize the importance of close clinical and radiological follow-up.
According to the literature, in selected patients with abdominal gunshot injury, non-operative 24-h observation is reported as applicable (17). In our study, there were four patients with luminal organ injury and they were classified as unsuccessful SNOM (Group 2). These patients were taken into surgery after $13 \mathrm{~h}$. In the evaluation of patients with regard to morbidity, surgical site infection occurred in only three patients. There was no complication after the repair of small bowel injuries. Colostomy treatment should also be performed if the patient is managed with operative treatment instead of SNOM; besides, we believe that no additional surgical risk occurred in SNOM of the colonic injury. Due to the small number of patients who failed SNOM, the discussion of this issue is limited.

In their meta-analysis covering the years 1990 to 2012, Lamb et al. (21) reported SNOM in 33\% of abdominal gunshot wounds, and reported a rate of $15.5 \%$ for delayed laparotomy. In our study, SNOM rate was $8.8 \%$ and the failure rate of SNOM was $22.2 \%$, a higher value than that reported in the literature. In our series, the rate of patients with SNOM was lower than that in the literature. Because all our patients were with abdominal gunshot injuries wounded in a battlefield and as our center is in a different country from the battlefield, our study group mostly comprised complicated trauma patients. In addition, nonoperative approach as primary treatment could be applied at a lower rate than that reported in the literature because of the regional socio-cultural characteristics.

Study of the relevant literature shows that centers that apply SNOM are high-volume and experienced trauma centers. These institutions are Level 1 trauma centers according to the trauma center classification of the American College of Surgeons and American Trauma Society $(24,25)$. Our center is between Levels 2 and 3. Our center is compatible with Level 2 in terms of its physical and technical facilities, but since we have no management protocol for trauma patients and cannot present a definitive treatment to all patients, our center nears Level 3. There are fewer studies carried out at low-volume trauma centers with limited experience $(6,26,27)$.

Studies on SNOM have been carried out at a limited number of centers, and the capacity of trauma surgeons to undertake this management approach is unknown. A study by Jansen et al. (28) showed that slightly more than half of the surgeons in trauma centers are experienced in SNOM and that most of the surgeons work at Level 1 trauma centers. There is no consensus on and no classification of SNOM application and there are known regional differences in the practice of SNOM (27). We think that creating clinical application protocols in trauma centers and applying diagnosis and therapy according to these protocols may decrease the failure rates of SNOM. Moreover, in view of current medicolegality, SNOM seems to cause a marked anxiety in surgeons (6). Our center did not have a remarkable experience in abdominal gunshot wounds in the past. However, with necessary attention current medical approaches were applied referring to the literature.

\section{Limitations}

Our study has several limitations. First of all, our study was a retrospective clinical study. Our patient number was lower than that reported in other series in literature. Also because of the limitations we mentioned above, the rate of SNOM was low in our study than that in the literature. 


\section{CONCLUSION}

In conclusion, SNOM is a feasible management method in the treatment of abdominal gunshot wounds, especially in patients with only solid organ injuries. It is not possible to predict the success of SNOM, in advance. The most sensitive point in taking this approach is the selection of appropriate patients. To decrease the morbidity and mortality in SNOM, patient selection and management should be performed carefully. In the presence of alarming symptoms, laparotomy should always be kept in mind.

Ethics Committee Approval: Authors declared that the research was conducted according to the principles of the World Medical Association Declaration of Helsinki "Ethical Principles for Medical Research Involving Human Subjects" (amended in October 2013).

Informed Consent: Written informed consent was obtained from patients who participated in this study.

Peer-review: Externally peer-reviewed.

Author Contributions: Concept - N.İ., O.Ü.; Design - N.I.., O.Ü., U.M.M.; Supervision - O.Ü., O.Z.Ö.; Resource - N.I.,, O.Ü.; Materials - U.M.M., M.Y.; Data Collection and/or Processing - N.I., U.M.M., M.Y.; Analysis and/or Interpretation - N.I, O.Z.Ö, M.Y.; Literature Search - O.Ü., O.Z.Ö., U.M.M.; Writing Manuscript - N.I., O.Ü.; Critical Reviews - O.Ü., U.M.M.

Conflict of Interest: No conflict of interest was declared by the authors.

Financial Disclosure: The authors declared that this study has received no financial support.

\section{REFERENCES}

1. Shaftan GW. Indications for operation in abdominal trauma. Am J Surg 1960; 99: 657-664. [CrossRef]

2. Hardaway RM III. Vietnam wound analysis. J Trauma 1978; 18: 635-642. [CrossRef]

3. McAlvanah MJ, Shaftan GW. Selective conservatism in penetrating abdominal wounds: a continuing reappraisal. J Trauma 1978; 18: 206-212. [CrossRef]

4. Shah R, Max MH, Flint LM Jr. Negative laparotomy: mortality and morbidity among 100 patients. Am Surg 1978; 44: 150-154.

5. Demetriades D, Rabinowitz B, Sofianos C. Non-operative management of penetrating liver injuries: a prospective study. $\mathrm{Br}$ J Surg 1986; 73: 736-737. [CrossRef]

6. Weigelt JA, Kingman RG. Complications of negative laparotomy for trauma. Am J Surg 1988; 156: 544-547. [CrossRef]

7. Renz BM, Feliciano DV. Unnecessary laparotomies for trauma: a prospective study of morbidity. J Trauma 1995; 38: 350-356. [CrossRef]

8. Renz BM, Feliciano DV. The length of hospital stay after an unnecessary laparotomy for trauma: a prospective study. J Trauma 1996; 40: 187-190. [CrossRef]

9. Sosa JL. Unnecessary laparotomies for trauma: a prospective study of morbidity. J Trauma 1995; 39: 397-398. [CrossRef]

10. Demetriades D, Hadjizacharia P, Constantinou C, Brown C, Inaba $K$, Rhee $P$, et al. Selective nonoperative management of penetrating abdominal solid organ injuries. Ann Surg 2006; 244: 620-628. [CrossRef]
11. Beekley AC, Blackbourne LH, Sebesta JA, McMullin N, Mullenix PS, Holcomb JB. Selective nonoperative management of penetrating torso injury from combat fragmentation wounds. J Trauma 2008; 64: 108-116. [CrossRef]

12. Como JJ, Bokhari F, Chiu WC, Duane TM, Holevar MR, Tandoh MA, et al. Practice management guidelines for selective nonoperative management of penetrating abdominal trauma. J Trauma 2010; 68: 721-733. [CrossRef]

13. Muckart DJJ, Abdool-Carim ATO, King B. Selective conservative management of abdominal gunshot wounds: A prospective study. Br J Surg 1990; 77: 652-655. [CrossRef]

14. Demetriades D, Charalambides D, Lakhoo M, Pantanowitz D. Gunshot wounds of the abdomen; Role of selective conservative management. Br J Surg 1991; 78: 220-222. [CrossRef]

15. Chmielewski GW, Nicholas JM, Dulchavsky SA, Diebel LN. Nonoperative management of gunshot wounds of the abdomen. Am Surg 1995; 61: 665-668.

16. Demetriades D, Velmahos G, Cornwell E 3rd, Berne TV, Cober S, Bhasin PS, et al. Selective nonoperative management of gunshot wounds of the anterior abdomen. Arch Surg 1997; 132: 178-183. [CrossRef]

17. Velmahos GC, Demetriades D, Foianini E, Tatevossian R, Cornwell EE 3rd, Asensio J, et al. A selective approach to the management of gunshot wounds to the back. Am J Surg 1997; 174: 342-346. [CrossRef]

18. Navsaria PH, Nicol AJ, Edu S, Gandhi R, Ball CG. Selective nonoperative management in 1106 patients with abdominal gunshot wounds: conclusions on safety, efficacy, and the role of selective CT imaging in a prospective single-center study. Ann Surg 2015; 261: 760-764. [CrossRef]

19. Singh N, Hardcastle TC. Selective non operative management of gunshot wounds to the abdomen: a collective review. Int Emerg Nurs 2015; 23: 22-31. [CrossRef]

20. Starling SV, Rodrigues Bde L, Martins MP, da Silva MS, Drumond DA. Non operative management of gunshot wounds on the right thoracoabdomen. Rev Col Bras Cir 2012; 39: 286-294. [CrossRef]

21. Lamb CM, Garner JP. Selective non-operative management of civilian gunshot wounds to the abdomen: a systematic review of the evidence. Injury 2014; 45: 659-666. [CrossRef]

22. DuBose J, Inaba K, Teixeira PG, Pepe A, Dunham MB, McKenney $M$. Selective non-operative management of solid organ injury following abdominal gunshot wounds. Injury 2007; 38: 10841090. [CrossRef]

23. Leenen LP. Abdominal trauma: from operative to nonoperative management. Injury 2009; 40: 62-68. [CrossRef]

24. Salim A, Velmahos GC. When to operate on abdominal gunshot wounds. Scand J Surg 2002; 91: 62-66. [CrossRef]

25. http://www.amtrauma.org/resources/trauma-categorization/index.aspx

26. Fikry K, Velmahos GC, Bramos A, Janjua S, de Moya M, King DR, et al. Successful selective nonoperative management of abdominal gunshot wounds despite low penetrating traumavolumes. Arch Surg 2011; 146: 528-532. [CrossRef]

27. Hope WW, Smith ST, Medieros B, Hughes KM, Kotwall CA, Clancy TV. Non-operative management in penetrating abdominal trauma: is it feasible at a Level II trauma center? J Emerg Med 2012; 43: 190-195. [CrossRef]

28. Jansen JO, Inaba K, Resnick S, Fraga GP, Starling SV, Rizoli SB, et al. Selective non-operative management of abdominal gunshot wounds: survey of practise. Injury 2013; 44: 639-644.[CrossRef] 